CLINICAL RESEARCH ARTICLE

\title{
Psychological risks to mother-infant bonding during the COVID-19 pandemic
}

\author{
Cindy H. Liu ${ }^{1,2,3}$, Sunah Hyun ${ }^{1,3}$, Leena Mittal ${ }^{2,3}$ and Carmina Erdei ${ }^{1,3}$ \\ (c) The Author(s), under exclusive licence to the International Pediatric Research Foundation, Inc 2021
}

BACKGROUND: The purpose of this study was to examine the association between mental health symptoms, along with psychological experiences and coronavirus disease 2019 (COVID-19) related concerns, and self-reported maternal-infant bonding experiences of postpartum women.

METHODS: Using data collected from May 19 to August 17, 2020, this cross-sectional online study assessed 429 women to better understand the impact of the COVID-19 pandemic on women during the postpartum period. Enrolled respondents were asked to participate in a 30-45-min online survey about COVID-19-related experiences, pregnancy, stress, and well-being.

RESULTS: Postpartum women's depressive symptoms were related to lower quality maternal-infant bonding, but the anxiety symptoms were not associated with bonding. Maternal self-efficacy, but not social support, was associated with mothers' higher quality of maternal-infant bonding. COVID-19-related grief was significantly associated with lower quality bonding. On the other hand, COVID-19-related health worries were associated with higher quality of maternal-infant bonding.

CONCLUSIONS: We describe potential psychological risk factors to maternal-infant bonding among postpartum women during the pandemic period. To best support the medical and psychological well-being of the mothers and infants, enhanced interdisciplinary partnerships among perinatal healthcare professionals involved in primary and/or specialty care is needed.

Pediatric Research (2022) 91:853-861; https://doi.org/10.1038/s41390-021-01751-9

\section{IMPACT:}

- Unique COVID-19-related health and grief concerns exist, with implications for maternal-infant bonding.

- Depression but not anxiety is associated with lower maternal-infant bonding.

- Caregiving confidence, but not social support, is associated with higher maternal-infant bonding.

- It is critical to screen for postpartum depression and COVID-19-related grief during maternal follow-up and pediatric visits.

- Study findings inform and prioritize pediatric interventions toward enhancing maternal-infant bonding during the COVID19 era.

\section{INTRODUCTION}

The postpartum period is a major transition for many women, given the psychological, social, and physiological challenges that take place during this time. ${ }^{1,2}$ The convergence of these changes increases the vulnerability for the onset or exacerbation of mental health problems among women. ${ }^{3}$ Indeed, $10-30 \%$ of women will suffer from depression during the postpartum period; ${ }^{4-6}$ postpartum anxiety estimates range from 13 to $40 \%,{ }^{4,7}$ in contrast to anxiety estimates from 3.8 to $25 \%$ in the general population. ${ }^{8}$ Poor mental health in the postpartum period can lead to mothers' difficulties in maternal-infant bonding, a relational experience between mother and baby that is foundational for later developmental and family outcomes. ${ }^{9-11}$

Accordingly, understanding how psychological experiences influence the quality of maternal-infant bonding is a major priority within the field of maternal and child health, and arguably a major concern during the coronavirus disease 2019 (COVID-19) pandemic given the perinatal experiences faced by women during this time. To date, over twenty-five million cases of COVID-19 infection have been documented within the United States. ${ }^{12}$ Although discussions have assessed the physical health risks of COVID-19 transmission between mothers and children, ${ }^{13,14}$ there remains little empirical data regarding the psychological health and well-being among postpartum mothers during the pandemic. ${ }^{15-17}$ Emerging work on the general population demonstrates a rise in depression and anxiety symptoms in the pandemic era. ${ }^{18-21}$ While women are already at risk of perinatal depression and anxiety, ${ }_{1}^{2-24}$ the pandemic has led to a significant increase in their psychological distress levels, ${ }^{25,26}$ with concerns that stress can spill over to affect families ${ }^{27}$ and in vulnerable process of mother-infant bonding and relationship building that occurs after birth. ${ }^{28}$ It is therefore important to consider the extent to which the psychological experiences during the COVID-19 pandemic are associated with maternal-infant bonding experiences among postpartum women.

\footnotetext{
${ }^{1}$ Department of Pediatric Newborn Medicine, Brigham and Women's Hospital, Boston, MA, USA. 'Department of Psychiatry, Brigham and Women's Hospital, Boston, MA, USA.
} ${ }^{3}$ Harvard Medical School, Boston, MA, USA. ${ }^{凶}$ email: chliu@bwh.harvard.edu 
It is well established that postpartum depression and depressive symptoms are associated with lower quality maternal-infant bonding. ${ }^{29-31}$ Relatively less research has been conducted on the effects of anxiety, with the existing literature showing somewhat mixed results. ${ }^{32}$ While prenatal and postpartum anxiety appear to interfere with sensitive maternal caregiving, ${ }^{33}$ postpartum anxiety has not necessarily been found to be associated with poorer mother-infant bonding. ${ }^{34}$ For instance, in some work, negative associations between maternal anxiety and bonding quality were no longer significant after controlling for depressive symptoms. ${ }^{31,32}$ Thus, symptoms of anxiety may lead to different effects on maternal-infant bonding than do symptoms of depression.

Maternal self-efficacy-the mother's belief or confidence in her ability to care for her baby-is a potential target for infant mental health interventions as it relates to multiple positive outcomes for both mothers and their babies. ${ }^{35}$ Studies show higher maternal self-efficacy to be related to higher levels of mothers' positive emotions, compatibility, and their devotion to the child, ${ }^{36,37}$ and lower postpartum depression, ${ }^{38,39}$ which in turn may promote maternal-infant bonding.

Social support is imperative for the physical, mental, and emotional well-being of mothers, ${ }^{40,41}$ and an important factor to consider in understanding maternal-infant bonding as an outcome. ${ }^{42}$ Social support protects against the adverse effects of stress, ${ }^{43}$ which can promote a better mother-child relationship. ${ }^{44,45}$ Low social support is associated with mothers' postpartum depression at 1 year after childbirth, ${ }^{46}$ which can reduce a mother's ability to bond with her child.

These risk and protective factors may be implicated with maternal-infant bonding during the COVID-19 pandemic, given concern about elevated mental health symptoms during this time. Postpartum social support, which is integral to the mental health of women, has been altered, given quarantine measures and limited access to healthcare providers and supportive family members for mothers. ${ }^{47}$ The ability to foster a strong maternal-infant bond during the pandemic might be even more dependent on the confidence that a mother has in her own capacity to care for her infant. Postpartum mothers may be concerned about COVID-19-related risks to their own health and their baby's health. They may experience grief, that is, a sense of loss from the inability to prepare or engage in joyful routines before and after birth (e.g., baby showers, visits with friends and family members to share and celebrate major life milestones). These new sources of concern may also affect a mother's ability to bond with her baby.

This study sought to explore the associations between mental health symptoms, along with psychological experiences and COVID-19-related concerns, and reported maternal-infant bonding experiences among postpartum women. The data were drawn from an online study referred to as the PEACE Study (Perinatal Experiences and COVID-19 Effects; www.peacestudy2020.com), which was launched in May 2020 to understand the mental health and well-being of pregnant and postpartum women within the US during the COVID-19 pandemic. This cross-sectional analysis focused on postpartum women who gave birth in the previous 6 months. We hypothesized that among postpartum women, lower levels of depression and anxiety symptoms, higher levels of self-efficacy and social support, and lower levels of COVID-19related health concerns and grief would be associated with more optimal maternal-infant bonding. An important goal for this work was to prioritize intervention targets for mothers and infants during the COVID-19 pandemic.

\section{METHODS \\ Participants}

This cross-sectional study used Wave 1 data collection $(N=429)$ from the PEACE study, which aimed to better understand the impact of the COVID-
19 pandemic on women during the pregnancy and postpartum period. The data were collected from May 19 to August 17, 2020. Eligible participants included women aged 18 years or older living in the US who were at least 13 weeks pregnant at the time of enrollment or gave birth to a live baby in the past 6 months. Participants were recruited nationally using several recruitment methods such as email list-servs, social media outlets, and word of mouth. Enrolled respondents were asked to participate in a $30-45-\mathrm{min}$ online survey about COVID-19-related experiences, pregnancy, stress, and well-being. The online survey embedded various attention checks and human verification to ensure data quality. The data were further inspected to detect any response irregularities. The present analysis focused on data provided by postpartum women. All procedures were approved by the Mass General Brigham Institutional Review Board.

\section{Measures}

Risk and protective factors

COVID-19-related health: Four items were obtained from the Coronavirus Health Impact Survey (CRISIS; ref. ${ }^{48}$ ) to assess the individual's worry about COVID-19 affecting one's health and the health of family and friends. Participants could indicate their concern about being infected themselves, or their friends and family being infected, and their own physical and mental health influenced by COVID-19 on a scale of 1 (not at all) to 5 (extremely). Cronbach's a for measure items was 0.85 , indicating good reliability. A sum score of these four items was calculated.

COVID-19-related grief: Using a seven-item measure, maternal feelings of grief related to lost experiences during the COVID-19 pandemic were assessed. Three items were adapted from the Inventory of Complicated Grief to capture grief symptoms, ${ }^{49}$ including those that captured participants' emotions, such as feeling stunned or dazed over what happened, feeling that life is empty, and feeling bitter over the loss of daily routines and activities in reference to the pandemic. Another three items assessed participants' feelings regarding lost experiences due to COVID-19, such as missing out on celebrating major life events, losing the support of family and friends due to social distancing, and losing vital resources (e.g., housing, mentorship, food access). Reported grief experiences using this 6-item grief scale have been associated with mental health symptoms among perinatal women, college students, ${ }^{50}$ and young adults; ${ }^{51}$ it has also been associated with mental health diagnoses, ${ }^{52}$ and sleep problems. ${ }^{53}$ For this current study, one item specific to the perinatal period was added to assess any sad feelings due to being unable to fully celebrate the pregnancy and/or birth of their child with loved ones. All items were rated on a five-point Likert-type scale, with $1=$ (strongly disagree) and $5=$ (strongly agree). The alpha coefficient for the overall scale was 0.78 , indicating acceptable reliability. A sum score of these seven items was calculated, with higher scores indicating participants' higher levels of grief.

Depression symptoms: To assess participants' severity of depression, the Center for Epidemiologic Studies-Depression (CES-D)'s self-report measure was used. ${ }^{54}$ The 20 -item scale assesses symptoms over the past 7 days using four response options: Rarely or none of the time ( $<1$ day); Some or a little of the time (1-2 days); Occasionally or a moderate amount of the time (3-4 days), and Most or all of the time (5-7 days). Examples of items on the measure include: (1) "I felt that I could not shake off the blues even with the help of my family or friends"; (2) "My sleep was restless"; and (3) "I felt that people disliked me." The total scores range from 0 to 60 , with high scores indicating greater depressive symptoms.

Anxiety symptoms: Participants' current anxiety symptoms were assessed using the Generalized Anxiety Disorder Scale (GAD-7; ref. ${ }^{55}$ ). This widely used seven-item self-report measure assessed the frequency of anxiety symptoms experienced in the past 2 weeks. The ratings were made on a scale of 0 (not at all) to 3 (nearly every day). Possible total score range from 0 to 21, with high scores indicating higher anxiety symptoms.

Maternal self-efficacy: Using the Maternal Self-Efficacy Scales (MSES; ref. $\left.{ }^{39}\right)$, participants' self-efficacy was assessed. The measure consists of ten items, which assesses maternal competencies related to discrete parenting tasks. Participants indicated how they currently feel about specific parenting tasks associated with infant care on a 4-point scale response option, ranging from 1 (not good at all/l don't understand my baby at all) 
to 4 (very good/l understand my baby almost all the time). The total score ranges from 10 to 40 , with higher scores reflecting higher maternal selfefficacy.

Social support: To assess participants' perception of social support, the Multidimensional Scale of Perceived Social Support (MSPSS) was used. ${ }^{56}$ This self-report measure includes 12 items. The ratings were made using a 1 (very strongly disagree) to 7 (very strongly agree) scale. Possible total scores range from 12 to 84 , with high scores indicating higher perceived social support.

\section{Outcomes}

Maternal-infant bonding: Participants' postnatal parental bonding with their baby was assessed using the Maternal Postpartum Attachment Scale (MPAS; ref. ${ }^{57}$ ). This 19 -item measure is divided into three factors. The first factor consists of nine items, in which respondents rate their confidence and satisfaction in interaction with the infant. The second factor consisted of five items and assessed participants' reflection of the absence of hostile feelings or anger toward the infant. The last factor consisted of five items that assessed participants' desire for physical closeness and joy in interaction with the baby. Each item had a two-, four-, or five-point scale response option, with higher scores reflecting postnatal bonding and lower scores suggesting a problematic mother-toinfant bond.

\section{Covariates}

Duration of pandemic: The number of days between the date when COVID-19 was declared as a pandemic in the US (March 13, 2020) and when each respondent started the survey was calculated in "days." This was included as a covariate given the possible correlation between the date of the survey administration and the outcome measures.

\section{Data analytic plan}

The variables were normally distributed, with predictors indicating acceptable levels of collinearity $(<5)$ with the exception of maternal race. As such, we report race characteristics as part of our descriptive data (Table 1), but do not incorporate race into the models. Beyond descriptive analyses on all variables, zero-order correlations were calculated for all variables, with significance set at $p<0.05$. Multiple regression analyses were conducted with maternal-infant bonding regressed on sociodemographic characteristics (Block 1), mental health symptoms (Block 2), psychosocial factors (Block 3), and COVID-19-related concerns (Block 4). We utilized SPSS 26.0 statistical software to perform analyses.

\section{RESULTS}

Table 1 provides descriptive characteristics of our sample. The mean age of participants was 33.7 years with the large majority of women being White and college-educated. Approximately $50 \%$ of respondents had a household income of over $\$ 150,000$ per year. Infants' average age was 12.6 weeks, with an equal distribution of males and females. This was the first pregnancy for $39.9 \%$ of women in this study. The overwhelming majority of participants had singleton births (98.6\%). Furthermore, $7.9 \%$ were born before 37 weeks of gestation and $10.5 \%$ required care in the neonatal intensive care unit (NICU). On average, participants completed the survey 112.9 days after the pandemic was declared a national emergency in the US (March 13, 2020).

Table 2 displays the mean scores for self-reported depression and anxiety symptoms as measured by standardized measures (CES-D, GAD-7). Participants had a mean score of $13.24(S D=8.96)$ on the CES-D and a mean score of $6.47(S D=5.06)$ on the GAD-7 (probable clinical depression and anxiety is indicated with CES-D $>16$ and GAD-7 > 10, respectively). Participants reported respective scores of $32.85(S D=4.13)$ and $68.78(S D=13.03)$ on the MSES and MSPSS. As a reference, scores between 30 and 40 on the MSES reflect responses such as "good enough" to "very good" and scores between 60 and 70 on the MSPSS refer to responses between "mildly agree" to "strongly agree" (a range that remains below "very strongly agree," the highest option on the scale). The mean scores for COVID-19-related concerns were $12.12(S D=3.37)$
Table 1. Demographic characteristics from Wave I of the PEACE Study, data collected between May 19 to August 17, 2020.

\begin{tabular}{|c|c|}
\hline Predictors & Means (range) or \% \\
\hline \multicolumn{2}{|l|}{ Infant sex } \\
\hline Male & $48.3 \%$ \\
\hline Female & $51.7 \%$ \\
\hline Infant age (weeks) & $12.6(0-30.0)$ \\
\hline Maternal age (years) & $33.7(20.0-44.0)$ \\
\hline \multicolumn{2}{|l|}{ Maternal race } \\
\hline White & $91.4 \%$ \\
\hline Black or African American & $0.5 \%$ \\
\hline Hispanic or Latino & $4.0 \%$ \\
\hline Asian and Pacific Islander & $4.2 \%$ \\
\hline Other & $0 \%$ \\
\hline \multicolumn{2}{|l|}{ Maternal education } \\
\hline Less than college & $6.8 \%$ \\
\hline College & $28.9 \%$ \\
\hline Masters & $44.3 \%$ \\
\hline Doctorate & $20.0 \%$ \\
\hline \multicolumn{2}{|l|}{ Household income (USD/year) } \\
\hline$<\$ 74,999$ & $10.8 \%$ \\
\hline$\$ 75,000-149,999$ & $38.6 \%$ \\
\hline$\$ 150,000-224,999$ & $28.5 \%$ \\
\hline$>\$ 225,000$ & $22.1 \%$ \\
\hline \multicolumn{2}{|l|}{ First pregnancy } \\
\hline No & $60.1 \%$ \\
\hline Yes & $39.9 \%$ \\
\hline \multicolumn{2}{|l|}{ Multiparity } \\
\hline No & $98.6 \%$ \\
\hline Yes & $1.4 \%$ \\
\hline \multicolumn{2}{|l|}{ NICU admissions } \\
\hline No & $89.5 \%$ \\
\hline Yes & $10.5 \%$ \\
\hline \multicolumn{2}{|l|}{ Premature (<37 weeks) } \\
\hline $\begin{array}{l}\text { No } \\
\text { Yes }\end{array}$ & $\begin{array}{l}92.1 \% \\
7.9 \%\end{array}$ \\
\hline Pandemic duration (days) & $112.9(69.0-150.0)$ \\
\hline
\end{tabular}

$$
N=429 \text {. }
$$

for health worries and $22.33(\mathrm{SD}=4.42)$ for grief experiences, respectively. Scores ranging from 10 to 15 on COVID-19-related health concerns reflect responses ranging from "slightly" to "moderately" in agreement on items. Scores from 20 to 30 on the COVID-19-related grief scale reflect responses that range from "disagree" to "agree," which encompass the middle range of the scale. Finally, participants had an average score of 78.82 (SD = 8.46) on the MPAS. Scores within 76-95 represent the range between the top two options on a five-point Likert scale; for instance, "very rarely" or "never" on items pertaining to negative perceptions of the baby.

Table 3 shows zero-order correlations of the variables. Mental health symptoms (depression, anxiety), psychosocial factors (social support, maternal self-efficacy), and COVID-19-related concerns (health worries, grief around lost experiences) were in general, significantly correlated in the expected directions, with higher social support and maternal self-efficacy associated with lower mental health symptoms and higher COVID-19-related concerns 


\section{6}

Table 2. Maternal mental health, COVID-related concerns, and maternal experiences from Wave I of the PEACE Study, data collected between May 19 to August 17, 2020.

\begin{tabular}{|lr|}
\hline $\begin{array}{l}\text { Target variables } \\
\text { Mental health symptoms }\end{array}$ & Means (SD) \\
\hline $\begin{array}{l}\text { Depression (CES-D) } \\
\text { Generalized anxiety (GAD-7) }\end{array}$ & $13.24(8.96)$ \\
\hline $\begin{array}{l}\text { Psychosocial factors } \\
\text { Social support (MSPSS) }\end{array}$ & $6.47(5.06)$ \\
\hline$\quad$ Maternal self-efficacy (MSES) & $68.78(13.03)$ \\
\hline COVID-19-related concerns & $32.85(4.13)$ \\
\hline$\quad$ Grief & \\
\hline Health & $22.33(4.42)$ \\
\hline Maternal bonding & $12.12(3.37)$ \\
\hline Attachment (MPAS) & $78.82(8.46)$ \\
\hline$N=429$. & \\
\hline
\end{tabular}

associated with higher mental health symptoms. Mothers who indicated that this was their first pregnancy, those who had younger aged infants, those with higher levels of reported depression and anxiety symptoms, and lower social support reported lower levels of maternal self-efficacy $(p<0.001)$. Higher levels of endorsed mental health symptoms and COVID-19-related grief were significantly associated with lower levels of maternal-infant bonding $(p<0.001)$, whereas higher social support and maternal self-efficacy were associated with higher levels of maternal-infant bonding $(p<0.001)$.

Table 4 reports the multiple regression results, with sociodemographic characteristics entered in Block 1, mental health symptoms (depression, anxiety) in Block 2, psychosocial factors (social support, maternal self-efficacy) in Block 3, and COVID-19related concerns (COVID-19-related health worries and grief) in Block 4. The change in $R^{2}$ for each block was significant $(p<0.05)$. Infant age appeared to be negatively associated with maternal-infant bonding $(\beta=-0.157, p<0.001)$. Furthermore, mothers holding a postgraduate degree were more likely than those with less than a college education to report lower maternal-infant bonding $(\beta=-0.166, p<0.05)$ and those with a household income of over US\$225,000 compared to those with an income $<$ US\$75,000 were more likely to report lower levels of maternal-infant bonding $(\beta=-0.091, p<0.01)$. First pregnancies were associated with higher levels of bonding $(\beta=0.105, p<0.01)$. After accounting for sociodemographic characteristics as well as the pandemic duration, higher levels of reported depression symptoms were associated with lower levels of maternal-infant bonding $(\beta=-0.376, p<0.001)$. No statistically significant associations were observed between anxiety symptoms and maternal-infant bonding ( $\beta=0.072, p=$ n.s.). After taking mental health symptoms into account, higher levels of maternal selfefficacy were correlated with higher levels of maternal-infant bonding $(\beta=0.480, p<0.001)$, with a similar trend observed in the association between social support with maternal-infant bonding $(\beta=0.073, p<0.1)$. Finally, both reported worries about health and grief specific to the COVID-19 pandemic uniquely contributed to the model above and beyond sociodemographic characteristics, mental health symptoms, and psychosocial factors. Higher levels of health worries were associated with higher levels of maternal-infant bonding $(\beta=0.093, p<0.05)$, whereas higher levels of grief were associated with lower levels of maternal-infant bonding $(\beta=-0.095, p<0.05)$. The predictors accounted for $55.2 \%$ of the model variance.

\section{DISCUSSION}

Maternal-infant bonding specifically refers to the emotional connectedness or bond experienced by the parent toward the infant $^{57}$ and is a concept considered highly significant in that a strong maternal bond likely facilitates the care of the infant in spite of the physical and emotional challenges involved in caregiving. ${ }^{58}$ The aim of our analysis was to identify and prioritize possible intervention targets in supporting maternal-infant bonding during the COVID-19 pandemic.

Our study yielded several new insights regarding risks to maternal-infant bonding among postpartum women during the first 6 months of the COVID-19 pandemic. First, depressive symptoms, but not anxiety symptoms were associated with lower ratings of maternal-infant bonding. Second, after accounting for these mental health symptoms, maternal self-efficacy, but not social support was related to higher levels of reported maternal-infant bonding. Finally, COVID-19-specific grief due to lost experiences during the pandemic was associated with lower levels of maternal-infant bonding, whereas COVID-19-related worries about health were associated with higher levels of maternal-infant bonding, after accounting for other predictors. Thus, our findings provide evidence that COVID-19 related concerns, particularly grief experiences, pose unique risks to the maternal-infant bonding process.

As observed in other studies, depressive symptoms were related to lower quality maternal-infant bonding. Mothers with depressive symptoms may face difficulties in developing affectionate regard for their infant with a limited range of positive emotions. This lack of a "maternal feeling" or the feeling of being "emotionally unavailable" may be attributed to depressive symptoms such as anhedonia, low, or dysregulated affect. ${ }^{59,60}$ The ability to bond with an infant may be affected in individuals who have a lowered positive future outlook, ${ }^{61}$ a perspective that may be particularly common for many during this pandemic. The possible escalating rates of depression within the population and the potential impact of depression on maternal-infant bonding from our study findings suggest that healthcare professionals must channel increased energy into addressing maternal depression during the COVID-19 pandemic.

It is notable that anxiety was not associated with maternal-infant bonding in our study; this finding appears to be consistent with prior published work. For instance, in a rural community sample, the expected negative associations between perinatal general anxiety and postpartum bonding were not found. ${ }^{29}$ Another study observed significant correlations between pregnancy-related fear and general anxiety with postpartum bonding, ${ }^{32}$ although the effects were no longer significant when accounting for depression. Similarly, mothers diagnosed with postpartum anxiety disorders in another study reported significantly lower maternal-infant bonding compared to healthy control mothers; ${ }^{31}$ however, the authors suggest that certain subclinical depressive features may explain this association. It is possible that anxiety does not interfere with the emotional connection that a mother might feel regarding the relationship she has with her infant; in other words, the mother's availability and capacity to bond with her infant may not depend on the anxiety experienced by the mother. Although anxious mothers have been observed to be less sensitive and responsive to their children $^{33,62}$ and are more likely to rate their children as being more temperamentally difficult, ${ }^{63}$ anxious mothers do not appear to show more negative affect towards their children compared to healthy control mothers. ${ }^{64}$ Tietz and colleagues have suggested that positive interactions with the infant might counterbalance any negative attitudes or behaviors due to an anxious predisposition, ${ }^{31}$ which, in turn, can further reinforce a mother's bond with her infant. While more work is needed to determine whether these findings hold for mothers with high levels of anxiety, our findings provide evidence that the risks between depression and 


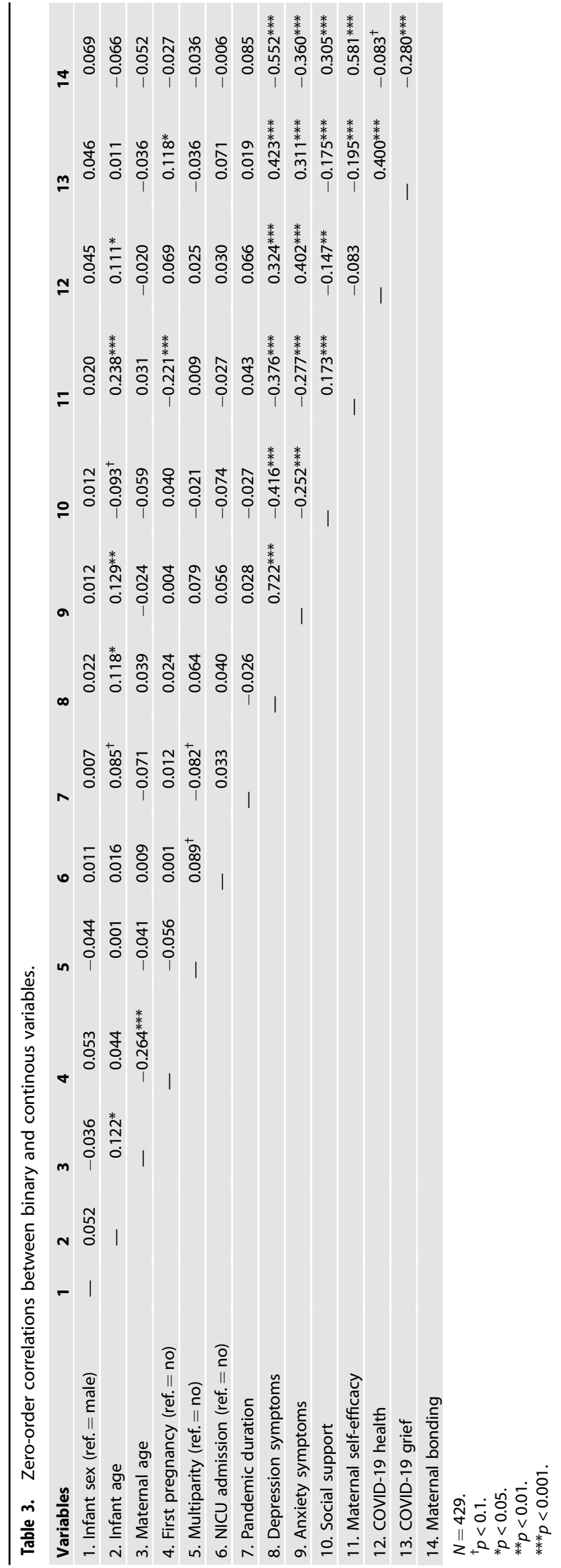

Table 4. Multiple regression predicting maternal bonding based on mental health symptoms, psychosocial factors, and COVID-19-related concerns.

\begin{tabular}{|c|c|c|c|}
\hline \multicolumn{4}{|l|}{ Total score } \\
\hline $\begin{array}{l}\text { Blocks of variables entered in } \\
\text { three steps }\end{array}$ & $\beta$ & $R^{2}$ & $\Delta R^{2}$ \\
\hline 1. Covariates & & 0.075 & $0.075^{* *}$ \\
\hline Infant sex (ref. $=$ male) & $0.071^{*}$ & & \\
\hline Infant age & $-0.157^{* * *}$ & & \\
\hline Maternal age & 0.026 & & \\
\hline \multicolumn{4}{|c|}{ Maternal education (ref. $=$ less than college) } \\
\hline College & -0.081 & & \\
\hline Masters & -0.074 & & \\
\hline Doctorate & $-0.166^{*}$ & & \\
\hline \multicolumn{4}{|c|}{ Household income (ref. $=<$ US\$74,999) } \\
\hline$\$ 75,000-149,999$ & 0.021 & & \\
\hline$\$ 150,000-224,999$ & $-0.060^{\dagger}$ & & \\
\hline$>\$ 225,000$ & $-0.091^{* *}$ & & \\
\hline First pregnancy (ref. $=$ no) & $0.105^{* *}$ & & \\
\hline Multiparity (ref. = no) & -0.016 & & \\
\hline NICU admission (ref. $=$ no) & 0.012 & & \\
\hline Pandemic duration & 0.043 & & \\
\hline 2. Mental health symptoms & & 0.370 & $0.295^{* * *}$ \\
\hline Depression & $-0.376^{* * *}$ & & \\
\hline Generalized anxiety & 0.072 & & \\
\hline 3. Psychosocial factors & & 0.539 & $0.168^{* * *}$ \\
\hline Social support & $0.073^{\dagger}$ & & \\
\hline Maternal self-efficacy & $0.480^{* * *}$ & & \\
\hline 4. COVID-19-related concerns & & 0.5478 & $0.010^{*}$ \\
\hline Grief & $-0.095^{*}$ & & \\
\hline Health & $0.093^{*}$ & & \\
\hline
\end{tabular}

$N=429$.

Model does not include maternal race due to multicollinearity.

${ }^{\dagger} p<0.1$.

${ }^{*} p<0.05$.

${ }^{* *} p<0.01$.

${ }^{* * *} p<0.001$.

anxiety symptoms are differentiated in their potential effects on maternal-infant bonding, even in the context of a pandemic that has elicited widespread stress and anxiety. Thus, interventions designed to ensure maternal-infant bonding should consider prioritizing depression in postpartum women at this time.

Contrary to expectations, maternal self-efficacy, but not social support, was associated with higher levels of maternal-infant bonding, despite prior work that underscores the role of social support on maternal-infant bonding ${ }^{65,66}$ and the current emphasis for increased social support and connection during the COVID19 pandemic. ${ }^{67,68}$ To date, there has been limited data on the association between maternal self-efficacy and maternal bonding. ${ }^{69}$ Those who feel confident in their parenting and caregiving abilities may have a greater capacity for being sensitive to infant needs. ${ }^{70}$ Similarly, these individuals may also feel more confident in fostering and establishing an attachment relationship, and thus experience a greater emotional connection with their infant. Maternal self-efficacy might be particularly relevant during the pandemic; mothers may need to rely more on their own abilities to care for their infant and/or to foster a connection with their infant. 
Social support may provide a source of relief for a mother, facilitate her adaptation to the new role as a mother, and allow her to be emotionally available to her infant. ${ }^{71}$ However, the association between social support and maternal-infant bonding in our study, while positive, did not achieve statistical significance. One possibility is that the social support assessed in our study, which focuses on emotional support, does not capture the instrumental support that may be more salient to the needs of postpartum mothers (e.g., someone to cook, watch other children in the home). It has been argued that social support may be particularly important for bonding among vulnerable populations $^{72}$ and this may not have been detected through our relatively low-risk sample. Although there is limited research on maternal social support and bonding, our findings appear to be consistent with the literature, with the majority of data under nonpandemic conditions showing weak to moderate associations. ${ }^{72}$ Altogether, more research is needed to understand how social support may play a role in the way mothers connect emotionally with their infants in general.

The concept of grief around lost experiences is a construct related to prior definitions of grief as previously studied, but with defining components that are perhaps unique to the COVID-19 era. People are known to highly value rituals around major life milestones across a variety of cultures-such as birth or reintegration of mothers and babies in the broader family context after birth-and they often desire to share their joy while expecting and having a baby with their partners and extended family. As COVID-19 infection prevention guidelines have reduced these opportunities, we sought to explore whether the loss of these major life celebratory experiences might be associated with experiences such as maternal-infant bonding. However, despite the recognition that there would many experiences of "loss" due to the pandemic, there was no COVID-19 related grief measure when we began this study, as our study took place a couple of months after the US lockdown (May 2020). To capture grief, we included items that spoke to the specific type of losses that women might experience due to the pandemic, as well as signs and symptoms of grief observed from other losses (e.g., "feeling stunned or dazed," "feeling that life is empty," and "feeling bitter").

Confirming our hypotheses, grief around lost experiences was significantly associated with lower mother-infant bonding. Although grief has shown to be associated with reported prenatal attachment, ${ }^{73}$ the large majority of work on maternal experiences of grief focuses on grief due to perinatal loss. ${ }^{74-77}$ There is also some literature on grief related to the loss of parenting a healthy child, including the grief that may be experienced by caregivers with children who have a disability. ${ }^{78}$ As well, parents of medically fragile infants who were admitted to the NICU may feel emotionally distant from their infants, ${ }^{79}$ as a result of their acute grief response to their child's medical diagnosis. ${ }^{80}$ Traumatic birth and non-vaginal births have also been associated with challenges in maternal bonding ${ }^{81-83}$ that may reflect some avoidance of the infant as they may serve as a reminder of the birth trauma. While grief was not directly assessed, previous work on widescale trauma found that mothers exposed to war trauma had lower levels of emotional availability. ${ }^{77}$ It is possible that grief reactions due to the pandemic, and the possibility of being overwhelmed by such feelings of grief may impede the mother's ability to be emotionally available to her baby.

Interestingly, COVID-19-related worries were associated with higher levels of maternal-infant bonding. Pregnancy is thought to enhance a mother's ability to establish an attachment relationship with her baby and to provide care. ${ }^{84,85}$ For some mothers, the pandemic itself may further elicit the need for the mother to protect her infant. Based on our findings, it is possible that mothers who worry a great deal about their health seem to also have the capacity to build a stronger emotional tie with their infant. Evidence that supports this interpretation is that high cortisol levels, which are usually associated with stress, ${ }^{86}$ have been shown to be elevated among mothers who are responsive or sympathetic to their newborn cries, ${ }^{87,88}$ suggesting that some level of worry, especially as it relates to the baby's well-being, may be necessary. While worry could reflect a mother's inclination to protect her infant, with primary maternal preoccupation being adaptive, ${ }^{89,90}$ the question arises whether being overly preoccupied about COVID-19 health risks could potentially go on to limit a mother's ability to connect with her infant in the longer term. As such, more research is needed to determine the extent to which COVID-19 worries about health may be adaptive or maladaptive for maternal-infant bonding over time. Either way, the COVID-19 pandemic is a new and major event for this generation and therefore a new source for health worries that may have implications for the way that mothers connect with their children.

We note that a number of sociodemographic variables were associated with maternal-infant bonding. Women who reported that this was their first pregnancy were more likely to report higher levels of bonding, as well as those with younger and female infants. It is likely that mothers without other children to care for may have more available time to reflect on their pregnancy and transition to parenthood, and therefore may be more connected with infants of their first pregnancy during the postpartum period. They may also experience less stress relative to those who have to provide care for other children as schools have been closed and childcare options have become limited. However, it is unclear how specific infant characteristics involving age and gender might promote higher maternal-infant bonding experiences; the literature on this is relatively sparse ${ }^{69}$ and warrants greater attention. Finally, mothers with a postgraduate degree compared to those with less than a college degree and those with a household income above US\$225,000 relative to those with an income under US\$75,000 reported lower levels of bonding. It is unclear why our data showed these associations. One possibility is that mothers who have higher levels of income or education may be more comfortable and/or face less stigma in disclosing the challenges they may face with caregiving or interacting with their infant. ${ }^{91,92}$ More research is needed to understand the maternal-infant bonding experiences among different socioeconomic status levels that are often thought to be low risk.

Several limitations must be addressed within the present study. First, since our results were derived from the cross-sectional data, no causal inference between our variables can be drawn. Second, the data presented here were obtained via self-report which has inherent limitations related to recall bias, and the standardized measures utilized in our survey while useful for screening for mental health symptoms are not diagnostic. Relatedly, we acknowledge the limitation in the use of the grief subscale as more work is needed to determine whether the signs and symptoms of grief during a pandemic reflect a factor distinct from symptoms of depression and anxiety ${ }^{93,94}$ and whether such experiences show long-term impairment. ${ }^{95-97}$ Third, in an effort to obtain a large number of respondents, participants in the study were recruited through convenience sampling. Our sample is also largely comprised of White and high socioeconomic status (SES) women. Study advertisements (social media advertisements, email list-servs), even those with targeted language are likely insufficient for engaging women of color and women at lower SES to participate in survey studies given various barriers to research participation. ${ }^{98}$ Given the disproportionate mental health burden placed on women of color within the pandemic, it is possible our findings do not reflect the wider range of experiences. It is also possible that the direction of effects could differ among these groups. Caution should be taken in generalizing findings to all postpartum women and their infants. 


\section{Clinical implications}

Evidence suggests that mother-infant bonding and the quality of the mother-infant relationship can have a significant impact on child neurodevelopment ${ }^{99}$ and overall family functioning; hence, it is critical to address existing problems early on. The findings in this study help inform and prioritize intervention targets to enhance maternal-infant bonding during the COVID-19 era; these should focus on the enhancement of both maternal mental health, as well as pediatric anticipatory guidance to achieve this goal. This calls for enhanced interdisciplinary partnerships among perinatal healthcare professionals involved in the primary and/or specialty care of both the mother and the infant, to best support the medical and psychological well-being of the family unit. Drawing on this data, specific considerations for healthcare professionals might include:

(1) Underscoring the importance of supporting and screening for postpartum depression during material follow-up visits and well-child visits as per the American Academy of Pediatrics' policy statement. ${ }^{100}$ As postpartum depression is now viewed as an adverse childhood experience with potential long-term implications for both mother and infant, there has been a recent call for multidisciplinary collaboration to screen and address this issue in pediatric settings in addition to maternal follow-up settings, to optimize the outcomes of the entire family. As such, primary pediatric healthcare professionals have been urged to take an active role in postpartum depression screening at $1,2,4$, and 6 months well-child visits; this call to action becomes even more salient during the COVID-19 pandemic when vulnerable populations are at increased risk of experiencing stress contagion.

(2) Focusing on dyadic interventions that increase maternal selfefficacy and confidence in parenting skills. These interventions often include encouraging and supporting meaningful interactions between mothers and infants, coaching parents on reading infant cues, and enhancing their own responsivity skills and connection with their infants. ${ }^{101-103}$ As these types of strategies benefit both hospitalized ${ }^{103}$ and healthy infants at home, evidence-based interventions that focus on healthy attachment and parent-child relationships should be prioritized and adapted as appropriate for each setting.

(3) Assessing the unique COVID-19-related health and grief concerns that each family unit might experience. Given our findings, there may be circumstances for which these experiences confer risk or indicate a feature of resilience within the family. For instance, worry about the infant may be warranted and normative and depending on the circumstances, should not be perceived as a risk factor. Providers and patient conversations are key to identifying the needs during the perinatal period; ${ }^{105}$ given the unique experiences faced by each family, understanding the circumstances of the caregivers can help to align and optimize resources for supporting the family.

\section{REFERENCES}

1. Brummelte, S. \& Galea, L. A. M. Postpartum depression: etiology, treatment and consequences for maternal care. Hormones Behav. 77, 153-166 (2016).

2. Tully, K. P., Stuebe, A. M. \& Verbiest, S. B. The fourth trimester: a critical transition period with unmet maternal health needs. Am. J. Obstet. Gynecol. 217, 37-41 (2017).

3. O'Hara, M. W. \& Wisner, K. L. Perinatal mental illness: definition, description and aetiology. Best. Pract. Res. Clin. Obstet. Gynaecol. 28, 3-12 (2014).

4. Falah-Hassani, K., Shiri, R. \& Dennis, C.-L. Prevalence and risk factors for comorbid postpartum depressive symptomatology and anxiety. J. Affect. Disord. 198, 142-147 (2016).

5. Gavin, N. I. et al. Perinatal depression: a systematic review of prevalence and incidence. Obstet. Gynecol. 106, 1071-1083 (2005).

6. Shorey, S. et al. Prevalence and incidence of postpartum depression among healthy mothers: a systematic review and meta-analysis. J. Psychiatr. Res. 104, 235-248 (2018).
7. Field, T. Postnatal anxiety prevalence, predictors and effects on development: a narrative review. Infant Behav. Dev. 51, 24-32 (2018).

8. Remes, O., Brayne, C., Linde, R. \& van der, Lafortune, L. A systematic review of reviews on the prevalence of anxiety disorders in adult populations. Brain Behav. 6, e00497 (2016).

9. Giallo, R., Woolhouse, H., Gartland, D., Hiscock, H. \& Brown, S. The emotional-behavioural functioning of children exposed to maternal depressive symptoms across pregnancy and early childhood: a prospective Australian pregnancy cohort study. Eur. Child Adolesc. Psychiatry 24, 1233-1244 (2015).

10. Slomian, J., Honvo, G., Emonts, P., Reginster, J.-Y. \& Bruyère, O. Consequences of maternal postpartum depression: a systematic review of maternal and infant outcomes. Women's Health 15, 1745506519844044 (2019).

11. Vafai, Y., Steinberg, J. R. \& Shenassa, E. D. Maternal postpartum depressive symptoms and infant externalizing and internalizing behaviors. Infant Behav. Dev. 42, 119-127 (2016).

12. Centers for Disease Control and Prevention. COVID data tracker [Internet]. https://covid.cdc.gov/covid-data-tracker (2020).

13. Alzamora, M. C. et al. Severe COVID-19 during pregnancy and possible vertical transmission. Am. J. Perinatol. 37, 861-865 (2020).

14. Centers for Disease Control and Prevention. Coronavirus disease 2019 (COVID19) [Internet]. https://www.cdc.gov/coronavirus/2019-ncov/daily-life-coping/ reducing-stigma.html (2020).

15. Liu, C. H., Erdei, C. \& Mittal, L. Risk factors for depression, anxiety, and PTSD symptoms in perinatal women during the COVID-19 pandemic. Psychiatry Res. 295, 113552 (2020).

16. Yan, H., Ding, Y. \& Guo, W. Mental health of pregnant and postpartum women during the coronavirus disease 2019 pandemic: a systematic review and metaanalysis. Front. Psychol. 11, 3324 (2020).

17. Hessami, K., Romanelli, C., Chiurazzi, M. \& Cozzolino, M. COVID-19 pandemic and maternal mental health: a systematic review and meta-analysis. J. Matern. Fetal Neonatal Med. 1-8. https://doi.org/10.1080/14767058.2020.1843155 (2020).

18. Fitzpatrick, K. M., Harris, C. \& Drawve, G. Living in the midst of fear: depressive symptomatology among US adults during the COVID-19 pandemic. Depress. Anxiety 37, 957-964 (2020).

19. Lebel, C., MacKinnon, A., Bagshawe, M., Tomfohr-Madsen, L. \& Giesbrecht, G. Elevated depression and anxiety symptoms among pregnant individuals during the COVID-19 pandemic. J. Affect. Disord. 277, 5-13 (2020).

20. Rajkumar, R. P. COVID-19 and mental health: a review of the existing literature. Asian J. Psychiatr. 52, 102066 (2020).

21. Wang, $C$. et al. A longitudinal study on the mental health of general population during the COVID-19 epidemic in China. Brain Behav. Immun. 67, 40-48 (2020).

22. Liu, C. H. \& Tronick, E. Re-conceptualising prenatal life stressors in predicting post-partum depression: cumulative-, specific-, and domain-specific approaches to calculating risk. Paediatr. Perinat. Epidemiol. 27, 481-490 (2013).

23. Liu, C. H., Giallo, R., Doan, S. N., Seidman, L. J. \& Tronick, E. Racial and ethnic differences in prenatal life stress and postpartum depression symptoms. Arch. Psychiatr. Nurs. 30, 7-12 (2016).

24. Leach, L. S., Poyser, C. \& Fairweather-Schmidt, K. Maternal perinatal anxiety: a review of prevalence and correlates. Clin. Psychologist 21, 4-19 (2017).

25. Davenport, M. H., Meyer, S., Meah, V. L., Strynadka, M. C. \& Khurana, R. Moms are not OK: COVID-19 and maternal mental health. Front. Glob. Women's Health 1, 1 (2020).

26. Zanardo, V. et al. Psychological impact of COVID-19 quarantine measures in northeastern Italy on mothers in the immediate postpartum period. Int. J. Gynecol. Obstet. 150, 184-188 (2020).

27. Liu, C. H. \& Doan, S. N. Psychosocial stress contagion in children and families during the COVID-19 pandemic. Clin. Pediatr. 59, 853-855 (2020).

28. Erdei, C. \& Liu, C. H. The downstream effects of COVID-19: a call for supporting family wellbeing in the NICU. J. Perinatol. 40, 1283-1285 (2020).

29. Edhborg, M., Nasreen, H.-E. \& Kabir, Z. N. Impact of postpartum depressive and anxiety symptoms on mothers' emotional tie to their infants 2-3 months postpartum: a population-based study from rural Bangladesh. Arch. Women's Ment. Health 14, 307-316 (2011)

30. Taylor, A., Atkins, R., Kumar, R., Adams, D. \& Glover, V. A new Mother-to-Infant Bonding Scale: links with early maternal mood. Arch. Women's Ment. Health 8, 45-51 (2005).

31. Tietz, A., Zietlow, A.-L. \& Reck, C. Maternal bonding in mothers with postpartum anxiety disorder: the crucial role of subclinical depressive symptoms and maternal avoidance behaviour. Arch. Women's Ment. Health 17, 433-442 (2014).

32. Dubber, S., Reck, C., Müller, M. \& Gawlik, S. Postpartum bonding: the role of perinatal depression, anxiety and maternal-fetal bonding during pregnancy. Arch. Women's Ment. Health 18, 187-195 (2015).

33. Feldman, R., Greenbaum, C. W., Mayes, L. C. \& Erlich, S. H. Change in motherinfant interactive behavior: relations to change in the mother, the infant, and the social context. Infant Behav. Dev. 20, 151-163 (1997). 
34. Edhborg, M., Seimyr, L., Lundh, W. \& Widström, A.-M. Fussy child - difficult parenthood? Comparisons between families with a "depressed" mother and non-depressed mother 2 months postpartum. J. Reprod. Infant Psychol. 18, 225-238 (2000).

35. Troutman, B., Moran, T. E., Arndt, S., Johnson, R. F. \& Chmielewski, M. Development of parenting self-efficacy in mothers of infants with high negative emotionality. Infant Ment. Health J. 33, 45-54 (2012).

36. Jones, T. L. \& Prinz, R. J. Potential roles of parental self-efficacy in parent and child adjustment: a review. Clin. Psychol. Rev. 25, 341-363 (2005).

37. Ruchala, P. L. \& James, D. C. Social support knowledge of infant development, and maternal confidence among adolescent and adult mothers. J. Obstet. Gynecol. Neonatal Nurs. 26, 685-689 (1997).

38. Law, K. H. et al. Stress, depressive symptoms, and maternal self-efficacy in firsttime mothers: modelling and predicting change across the first six months of motherhood. Appl. Psychol. Health Well-Being 11, 126-147 (2019).

39. Teti, D. M. \& Gelfand, D. M. Behavioral competence among mothers of infants in the first year: the mediational role of maternal self-efficacy. Child Dev. 62, 918-929 (1991).

40. DeSousa, M., Reeve, C. L. \& Peterman, A. H. Development and initial validation of the Perceived Scarcity Scale. Stress Health 36, 131-146 (2020).

41. Razurel, C., Kaiser, B., Sellenet, C. \& Epiney, M. Relation between perceived stress, social support, and coping strategies and maternal well-being: a review of the literature. Women health 53, 74-99 (2013).

42. Crockenberg, S. B. Infant irritability, mother responsiveness, and social support influences on the security of infant-mother attachment. Child Dev. 52, 857-865 (1981).

43. Taylor, S. E. In The Oxford Handbook of Health Psychology (ed. Friedman, H. S.) 189-214 (Oxford University Press, 2011).

44. Guajardo, N. R., Snyder, G. \& Petersen, R. Relationships among parenting practices, parental stress, child behaviour, and children's social-cognitive development. Infant Child Dev. 18, 37-60 (2009).

45. Moss, E., Rousseau, D., Parent, S., St-Laurent, D. \& Saintonge, J. Correlates of attachment at school age: maternal reported stress, mother-child interaction, and behavior problems. Child Dev. 69, 1390-1405 (1998).

46. Ganann, R., Sword, W., Thabane, L., Newbold, B. \& Black, M. Predictors of postpartum depression among immigrant women in the year after childbirth. $J$. Women's Health 25, 155-165 (2016).

47. Khoury, J. E., Atkinson, L., Bennett, T., Jack, S. M. \& Gonzalez, A. COVID-19 and mental health during pregnancy: the importance of cognitive appraisal and social support. J. Affect. Disord. 282, 1161-1169 (2021).

48. Merikangas, K., Milham, M. \& Stringaris, A. The Coronavirus Health Impact Survey (CRISIS) [Internet]. http://www.crisissurvey.org/ (2020).

49. Prigerson, H. G. et al. Inventory of complicated grief: a scale to measure maladaptive symptoms of loss. Psychiatry Res. 59, 65-79 (1995)

50. Conrad, R. C., Koire, A., Pinder-Amaker, S. \& Liu, C. H. College student mental health risks during the COVID-19 pandemic: implications of campus relocation. J. Psychiatr. Res. 136, 117-126 (2021).

51. Liu, C. H., Zhang, E., Wong, G. T. F., Hyun, S. \& Hahm, H. C. Factors associated with depression, anxiety, and PTSD symptomatology during the COVID-19 pandemic: clinical implications for U.S. Young Adult Mental Health. Psychiatry Res. 290, 113172 (2020).

52. Liu, C. H., Stevens, C., Conrad, R. \& Hahm, H. C. Evidence for elevated psychiatric distress, poor sleep, and quality of life concerns during the COVID-19 pandemic among U.S. young adults with suspected and reported psychiatric diagnoses. Psychiatry Res. 292, 113345 (2020).

53. Hyun, S., Hahm, H. C., Wong, G. T. F., Zhang, E. \& Liu, C. H. Psychological correlates of poor sleep quality among US young adults during the COVID-19 pandemic. Sleep Med. 78, 51-56 (2021).

54. Radloff, L. S. The CES-D Scale: a self-report depression scale for research in the general population. Appl. Psychol. Meas. 1, 385-401 (1977).

55. Spitzer, R. L., Kroenke, K., Williams, J. B. \& Löwe, B. A brief measure for assessing generalized anxiety disorder: the GAD-7. Arch. Intern. Med. 166, 1092-1097 (2006)

56. Zimet, G. D., Dahlem, N. W., Zimet, S. G. \& Farley, G. K. The multidimensional scale of perceived social support. J. Pers. Assess. 52, 30-41 (1988).

57. Condon, J. T. \& Corkindale, C. J. The assessment of parent-to-infant attachment: development of a self-report questionnaire. J. Reprod. Infant Psychol. 16, 57-57 (1998).

58. Kennell, J. \& McGrath, S. Starting the process of mother-infant bonding. Acta Paediatr. 94, 775-777 (2005).

59. Moehler, E., Brunner, R., Wiebel, A., Reck, C. \& Resch, F. Maternal depressive symptoms in the postnatal period are associated with long-term impairment of mother-child bonding. Arch. Women's Ment. Health 9, 273-278 (2006)
60. Nonnenmacher, N., Noe, D., Ehrenthal, J. C. \& Reck, C. Postpartum bonding: the impact of maternal depression and adult attachment style. Arch. Women's Ment. Health 19, 927-935 (2016).

61. Rossen, L. et al. Predictors of postnatal mother-infant bonding: the role of antenatal bonding, maternal substance use and mental health. Arch. Women's Ment. Health 19, 609-622 (2016).

62. Nicol-Harper, R., Harvey, A. G. \& Stein, A. Interactions between mothers and infants: Impact of maternal anxiety. Infant Behav. Dev. 30, 161-167 (2007).

63. Britton, J. R. Infant temperament and maternal anxiety and depressed mood in the early postpartum period. Women Health 51, 55-71 (2011).

64. Kaitz, M., Maytal, H. R., Devor, N., Bergman, L. \& Mankuta, D. Maternal anxiety, mother-infant interactions, and infants' response to challenge. Infant Behav. Dev. 33, 136-148 (2010).

65. Badr, L. K., Ayvazian, N., Lameh, S. \& Charafeddine, L. Is the effect of postpartum depression on mother-infant bonding universal? Infant Behav. Dev. 51, 15-23 (2018).

66. Ohara, M. et al. Social support helps protect against perinatal bonding failure and depression among mothers: a prospective cohort study. Sci. Rep. 7, 9546 (2017).

67. Lakshmin, P. Experts fear increase in postpartum mood and anxiety disorders [Internet]. The New York Times. https://www.nytimes.com/2020/05/27/parenting/ coronavirus-postpartum-depression-anxiety.html (2020).

68. Holohan, M. Pandemic isolation is leading to more postpartum depression, anxiety [Internet]. TODAY.com. https://www.today.com/parents/covid-19-pandemic-leadsmore-postpartum-depression-t179221 (2020).

69. Delavari, M., Mohammad-Alizadeh-Charandabi, S. \& Mirghafurvand, M. The relationship between maternal-fetal attachment and maternal self-efficacy in Iranian women: a prospective study. J. Reprod. Infant Psychol. 36, 302-311 (2018).

70. Leerkes, E. M. \& Crockenberg, S. C. The development of maternal self-efficacy and its impact on maternal behavior. Infancy 3, 227-247 (2002).

71. Kinsey, C. B., Baptiste-Roberts, K., Zhu, J. \& Kjerulff, K. H. Birth-related, psychosocial, and emotional correlates of positive maternal-infant bonding in a cohort of first-time mothers. Midwifery 30, e188-e194 (2014).

72. Tichelman, E. et al. Correlates of prenatal and postnatal mother-to-infant bonding quality: a systematic review. PLoS ONE 14, e0222998 (2019).

73. Gaudet, C., Séjourné, N., Camborieux, L., Rogers, R. \& Chabrol, H. Pregnancy after perinatal loss: association of grief, anxiety and attachment. J. Reprod. Infant Psychol. 28, 240-251 (2010).

74. Hutti, M. H. et al. Predicting grief intensity after recent perinatal loss. J. Psychosom. Res. 101, 128-134 (2017).

75. Markin, R. D. \& Zilcha-Mano, S. Cultural processes in psychotherapy for perinatal loss: breaking the cultural taboo against perinatal grief. Psychotherapy 55, 20 (2018).

76. Tseng, Y.-F., Cheng, H.-R., Chen, Y.-P., Yang, S.-F. \& Cheng, P.-T. Grief reactions of couples to perinatal loss: a one-year prospective follow-up. J. Clin. Nurs. 26, 5133-5142 (2017).

77. Punamäki, R.-L., Isosävi, S., Qouta, S. R., Kuittinen, S. \& Diab, S. Y. War trauma and maternal-fetal attachment predicting maternal mental health, infant development, and dyadic interaction in Palestinian families. Attach. Hum. Dev. 19, 463-486 (2017).

78. Brown, J. M. Recurrent grief in mothering a child with an intellectual disability to adulthood: grieving is the healing. Child Fam. Soc. Work 21, 113-122 (2016).

79. MacKay, L., Benzies, K., Barnard, C. \& Raffin Bouchal, S. Parental experiences caring for their hospitalized medically fragile infants: a description of grief, stress, and coping. Can. J. Nurs. Res. 0844562120954125 (2020).

80. Skelton, H., Dahlen, H. G., Psaila, K. \& Schmied, V. Facilitating closeness between babies with congenital abnormalities and their parents in the NICU: a qualitative study of neonatal nurses' experiences. J. Clin. Nurs. 28, 2979-2989 (2019).

81. Van Reenen, S. L., Van \& Rensburg, E. The influence of an unplanned caesarean section on initial mother-infant bonding: mothers' subjective experiences. J. Psychol. Afr. 23, 269-274 (2013).

82. Ayers, S., Eagle, A. \& Waring, H. The effects of childbirth-related post-traumatic stress disorder on women and their relationships: a qualitative study. Psychol. Health Med. 11, 389-398 (2006).

83. Stuijfzand, S., Garthus-Niegel, S. \& Horsch, A. Parental birth-related PTSD symptoms and bonding in the early postpartum period: a prospective population-based cohort study. Front. Psychiatry 11, 570727 (2020).

84. Bowlby, J. Attachment and Loss, Vol. 1: Attachment (Basic Books, 1969).

85. Cassidy, J. in Handbook of Attachment: Theory, Research, and Clinical Applications (eds Cassidy, J. \& Shaver, P. R.) 3-22 (The Guilford Press, 2008).

86. Liu, C. H. \& Doan, S. N. Innovations in biological assessments of chronic stress through hair and nail cortisol: conceptual, developmental, and methodological issues. Dev. Psychobiol. 61, 465-476 (2019). 
87. Giardino, J., Gonzalez, A., Steiner, M. \& Fleming, A. S. Effects of motherhood on physiological and subjective responses to infant cries in teenage mothers: a comparison with non-mothers and adult mothers. Hormones Behav. 53, 149-158 (2008).

88. Stallings, J., Fleming, A. S., Corter, C., Worthman, C. \& Steiner, M. The effects of infant cries and odors on sympathy, cortisol, and autonomic responses in new mothers and nonpostpartum women. Parent. Sci. Pract. 1, 71-100 (2001).

89. Hall, R. A. S. et al. Maternal psychological distress after preterm birth: disruptive or adaptive? Infant Behav. Dev. 49, 272-280 (2017).

90. Winnicott, D. W. in The Maternal Lineage: Identification, Desire, and Transgenerational Issues (ed. Mariotti, P.) 59-66 (Routledge, 2012).

91. Forder, P. M. et al. Honesty and comfort levels in mothers when screened for perinatal depression and anxiety. Women Birth 33, e142-e150 (2020).

92. Maxwell, D., Robinson, S. R. \& Rogers, K. "I keep it to myself": a qualitative metainterpretive synthesis of experiences of postpartum depression among marginalised women. Health Soc. Care Community 27, e23-e36 (2019).

93. Boelen, P. A. \& van den Bout, J. Complicated grief, depression, and anxiety as distinct postloss syndromes: a confirmatory factor analysis study. Am. J. Psychiatry 162, 2175-2177 (2005).

94. Dillen, L., Fontaine, J. R. \& Verhofstadt-Denève, L. Confirming the distinctiveness of complicated grief from depression and anxiety among adolescents. Death Stud. 33, 437-461 (2009).

95. Boelen, P. A. \& Prigerson, H. G. The influence of symptoms of prolonged grief disorder, depression, and anxiety on quality of life among bereaved adults. Eur. Arch. Psychiatry Clin. Neurosci. 257, 444-452 (2007).

96. Zhai, Y. \& Du, X. Loss and grief amidst COVID-19: a path to adaptation and resilience. Brain Behav. Immun. 87, 80-81 (2020).

97. Kokou-Kpolou, C. K., Fernández-Alcántara, M. \& Cénat, J. M. Prolonged grief related to COVID-19 deaths: do we have to fear a steep rise in traumatic and disenfranchised griefs? Psychol. Trauma 12, S94 (2020).

98. Hughes, T. B., Varma, V. R., Pettigrew, C. \& Albert, M. S. African Americans and clinical research: evidence concerning barriers and facilitators to participation and recruitment recommendations. Gerontologist 57, 348-358 (2017).

99. Le Bas, G. A. et al. The role of antenatal and postnatal maternal bonding in infant development: a systematic review and meta-analysis. Soc. Dev. 29, 3-20 (2020).

100. Earls, M. F., Yogman, M. W., Mattson, G. \& Rafferty, J. Committee on Psychosocia Aspects of Child and Family Health. Incorporating recognition and management of perinatal depression into pediatric practice. Pediatrics 143, e20183259 (2019).

101. Mihelic, M., Morawska, A. \& Filus, A. Effects of early parenting interventions on parents and infants: a meta-analytic review. J. Child Fam. Stud. 26, 1507-1526 (2017).

102. Benzies, K. M., Magill-Evans, J. E., Hayden, K. A. \& Ballantyne, M. Key components of early intervention programs for preterm infants and their parents: a systematic review and meta-analysis. BMC Pregnancy Childbirth 13, S10 (2013).
103. Beebe, B. et al. Family nurture intervention for preterm infants facilitates positive mother-infant face-to-face engagement at 4 months. Dev. Psychol. 54 2016-2031 (2018)

104. Paris, R., Bolton, R. E. \& Spielman, E. Evaluating a home-based dyadic intervention: changes in postpartum depression, maternal perceptions, and mother-infant interactions. Infant Ment. Health J. 32, 319-338 (2011).

105. Liu, C. H. \& Tronick, E. Do patient characteristics, prenatal care setting, and method of payment matter when it comes to provider-patient conversations on perinatal mood? Matern. Child Health J. 16, 1102-1112 (2012).

\section{AUTHOR CONTRIBUTIONS}

C.H.L. participated in the planning, design of the study, and analyzing the data. C.H.L., L.M., C.E., and S.H. participated in the acquisition of the data and interpretation of data. C.H.L. drafted the article for important intellectual content, and all authors participated in editing the manuscript. C.H.L. had primary responsibility for the final content. All authors read and approved the final manuscript.

\section{FUNDING INFORMATION}

Support for this manuscript was provided through the Mary A. Tynan Faculty Fellowship, the Family Health and Resilience Research Fund, the Weinberg and Barton Families, and an NIH K23 MH 107714-01 A1 award (to C.H.L.) and NIH T32 MH016259 (to S.H.)

\section{COMPETING INTERESTS}

The authors declare no competing interests.

\section{CONSENT STATEMENT}

Human subjects were consented prior to their participation.

\section{ADDITIONAL INFORMATION}

Correspondence and requests for materials should be addressed to Cindy $\mathrm{H}$. Liu.

Reprints and permission information is available at http://www.nature.com/ reprints

Publisher's note Springer Nature remains neutral with regard to jurisdictional claims in published maps and institutional affiliations. 